Article

\title{
Backward Reasoning and Epistemic Actions in Discovering Processes of Strategic Games Problems
}

\author{
Marta Barbero ${ }^{1,2, *(\mathbb{D})}$, Inés M. Gómez-Chacón ${ }^{3, * \mathbb{D}}$ and Ferdinando Arzarello ${ }^{2}$ (D) \\ 1 Facultad de Ciencias Matemáticas, Universidad Complutense de Madrid, 28040 Madrid, Spain \\ 2 Dipartimento di Matematica “G. Peano”, Università degli Studi di Torino, 20123 Turin, Italy; \\ ferdinando.arzarello@unito.it \\ 3 Instituto de Matemática Interdisciplinar, Facultad de Ciencias Matemáticas, \\ Universidad Complutense de Madrid, 28040 Madrid, Spain \\ * Correspondence: mabarb01@ucm.es (M.B.); igomezchacon@mat.ucm.es (I.M.G.-C.)
}

Received: 6 May 2020; Accepted: 15 June 2020; Published: 17 June 2020

\begin{abstract}
The paper focuses on the cognitive and epistemic characterization of backward reasoning in strategy games resolution. It explores the use of abstraction in context (AiC) theory as a tool for the analysis of the epistemic actions involved in these processes. We follow a qualitative methodology based on design experiments, focusing on a study carried out with mathematics and engineering students. The analysis allowed us to combine the two models derived from the epistemology of mathematical backward reasoning and AiC theory in a unified framework that allowed us to focus both short-term and long-term processes in mathematical activities. Recurring patterns of epistemic actions were identified.
\end{abstract}

Keywords: reasoning and proof; strategy games; backward reasoning; engineering education; mathematical education; abstraction processes; epistemic actions

\section{Introduction}

One of the most used reasoning modalities in problem solving and programming methods is that of mathematical backward reasoning, which is also a critical issue in advanced mathematics education. The growing interest of game-based university education was also one of the foundations of this research.

Mathematical backward reasoning is a modality of mathematical thinking involved in the method of analysis. It is used in problem solving discovering phases, and it consists of developing a series of logical steps beginning from the claim of the problem toward its premises, proceeding through logical correspondences [1]. It corresponds to what Lakatos calls the "retransmission of falsity" in mathematics reasonings. Lakatos distinguishes it from that used in what he calls Euclidean systems, where the transmission of truth goes from the set of axioms "downwards" to the rest of the system. In the latter, the logic behind is an instrument of proof; in the former, it can be considered as an organon of criticism [2] (p. 29). Both are necessary ingredients of the mathematical reasoning developed in the production of proofs and have been formalized within mathematics in the works of Hintikka and his school $[1,3]$.

In this research carried out with 185 mathematics and engineering university students at Spanish and Italian universities, the processes of backward reasoning were examined in order to provide a useful tool that could be employed for the development of teaching techniques suitable for the growing need for technological skills. As an example, with a diagnostic tool like the one developed through this study, we consider it possible to mechanize a certain machine learning algorithm (e.g., a meta-learning algorithm [4]) or an automatic approach, finding a more general procedure to solve problems. 
Through the study and design of new systems that incorporate cognitive and affective components, new technologies that better address human needs are developed [5-8]. Some investigators have tried to model and simulate human reasoning processes [6,9] with the objective to reproduce human behavior. The research presented here is a significant contribution to these studies.

A backward reasoning study can be used to improve students' achievement helping them to develop mathematical argumentation, inquiry, and proof processes. It plays a central role at the university level, where the encountered concepts are more abstract and require advanced mathematical thinking, particularly those associated with technology. The application of this type of reasoning is not intuitive: several authors have underlined the difficulties in using and understanding it as a general procedure $[10,11]$.

In previous studies analyzing mathematics and engineering students' thinking involved in problem solving activities [12,13], it was noted that the backward reasoning, as an emerging key process in the dialectics between inference processes, develops mainly in interrogative movements and is responsible for the generation of new ideas and elements in the solution process. This reasoning is used in its character of "ordering device:" Through it, the students manage to find elements necessary for the construction/definition of the objective and to connect more intuitive aspects.

We found that it is more difficult to reason backwards than forwards. That is, proceeding from the premises towards the end of the problem is easier. Thus, it is necessary to offer students a larger class of problems for which the backward reasoning is appropriate, such as the strategy games presented here. We also identified some factors in the cognitive and affect interplay that would inevitably cause difficulties for students. A previous investigation [14] showed how the epistemic emotions continually exert numerous operator effects, both linear and nonlinear, on attentional activity and on the ability to perceive goal-path obstacles and to overcome them. Understanding is linked with the appraisal of their ability to influence, with their ability to predict, and with mental flexibility [13,14]. The detected taxonomy of obstacles suggests that the lecturer, as a mediator of knowledge, explicitly takes the nature of backward reasoning underlying the interplay between cognitive and epistemological models. into account

This paper focuses on the cognitive and epistemic characterization of backward reasoning in strategy games resolution. In strategy games, players must make strategic choices to make their moves, thus allowing for a natural development of backward reasoning.

We use the words epistemic and cognitive to highlight two concurrent aspects of mathematical thinking: the formal aspects (axioms, proofs, theorems), which many times are intertwined with their algorithmic aspects (the algorithm of Euclidean division), and what many authors call the intuitive ways of mathematical reasoning, which are linked with the mental functions that allow one to elaborate and/or grasp the more formal aspects. Such two intertwined aspects of mathematical thinking have been pointed out both by mathematicians and by mathematics educators. The book of Hilbert and Cohn-Vossen [15] started as follows: "In mathematics, as in all scientific research, we find two tendencies: the tendency to abstraction-it seeks to work out (herauszuarbeiten) the logical points of view from the manifold material and bring this into systematic connection-and the other tendency, that of intuitive understanding (anschaulichkeit), which rather gets (ausgeht) the object and its substantive relationships from a living grasping." (p. XVII).

In this study, we explored the use of the (recognizing, building-with, and constructing) RBC model, as derived from abstraction in context (AiC) theory [16], which is described in Section 2.3. It is shown not only its relevance but also the need to extend it through other specific categories of backward reasoning (see Section 2.1) in order to analyze the epistemic actions involved in students' thoughts processes.

\section{Theoretical Elements}

This section is divided into three parts, the first dealing with the concepts of backward reasoning, the second with that of epistemic actions, and the third with AiC theory. 


\subsection{Backward Reasoning}

Forward reasoning alone is not exhaustive to fulfil the tasks of solving problems. Mathematicians like Pappus, Descartes, and Leibniz emphasized this fact [11]. Backward reasoning is known by different denominations: regressive analysis, backward solution, method of analysis, etc. It is the practice that involves the making of a number of arguments from the bottom of the problem and proceeds through logical correspondences that allow for the obtainment of something known or to be reached through other paths. This process includes different problem solving strategies: backtracking heuristics, reductio ad absurdum, starting with the end of the problem, and assuming the problem solved [17].

Pappus has contributed substantially to the clarification and exemplification of the method. In the seventh book of his Collection, he dealt with heuristics methods to solve the problems. He exemplified the method of analysis and synthesis, making the development of this reasoning clearer. Pappus defined the method of analysis as follows: "In analysis, we start from what is required, we take it for granted; and we draw correspondence from it and correspondence from the correspondence, till we reach a point that we can use as a starting point in synthesis. That is to say, in analysis we assume what is sought as already found (what we have to prove as true)." (authors' re-elaboration: [1] (p. 8) and [18] (p. 142)). Subsequently he pointed out: "This procedure we call analysis, or solution backward, or regressive reasoning." [1] (p. 8). Later, he defined the method of synthesis: "In synthesis, on the other hand, we suppose that which was reached last in analysis to be already done, and arranging in their natural order as consequents the former antecedents and linking them one with another, we in the end arrive at the construction of the thing sought. This procedure we call synthesis, or constructive solution, or progressive reasoning" [1] (pp. 8-9).

There is no analysis method without the synthesis one. Peckhaus [11] studied this analysis-synthesis scheme and affirmed that "The analytical [is] [...] the procedure which starts with the formulation of the problem and ends with the determination of the conditions for its solution. The synthetical represents the way from the conditions to the actual solution of the problem. [...] This branch of the scheme is deeply connected with the complementary [one]." Not only can analysis not exist without synthesis, but "synthesis cannot be isolated and presupposes analysis."

The concept of backward reasoning is an essential part of the analysis method and is strongly bounded to forward processes, as pointed out in Pappus' synthesis definition. Philosophers and mathematicians, from the ancient Greeks to now, have studied the characteristics of this type of reasoning. The main features that we found in literature are the following:

- Direction vs. cause-effect: In Pappus' definition, the backward direction of reasoning is highlighted. By applying the method, the premises of a certain idea are sought. In the 17th and 18th centuries, authors such as Arnauld and Nicole interpreted the method as a backward search for cause-effect relationships between ideas. By these, the connection between the notions in background and the problem can be identified [11,17].

- Breakdown: According to Plato and Pappus, this kind of reasoning allows for the reduction of the problem to its simplest components. The properties that define the assignment and the relationships between the objects involved in it are identified by extracting and investigating the principles that are at the base of the task. Aristotle, for example, underlined the fact that "sometimes, to solve a geometrical problem, you can only analyze a figure," breaking it down into its basic components and understanding the different parts of it [17].

- Introduction of auxiliary elements: Kant, Polya, and Hintikka focused their attention on a fundamental part of the process: the introduction of new elements (a well know practice in geometry with the auxiliary constructions). In progressive and deductive processes, all the bases are given, and from these, consequences are elaborated. Instead, in backward reasoning new notions appear and develop throughout the resolution according to the needs of the solver $[1,17]$. 
These categories, supported by the epistemology of mathematical knowledge and highlighted by mathematicians, were considered in this study as the major key to analyze backward reasoning in learning processes.

\subsection{Epistemic Actions}

The concept of epistemic action was introduced into cognitive sciences by Kirsh and Maglio [19] to indicate actions that facilitate cognition and allow for problems to be solved more quickly. These actions help to acquire useful information for the resolution. This information is hidden or difficult to compute mentally: Epistemic actions have the purpose to simplify the mental processes to reach it. In mathematics education, the term was first used by Hershkowitz, Schwarz, and Dreyfus [20] in their research on abstraction, which they derived from Pontecorvo and Girardet [21]. The mental processes that occur in the student while solving a problem are not directly observable but can be identified through the analysis of the students' utterances and of their actions. Epistemic actions allow for the identification of the mental progresses in which knowledge is used or built and to operationally describe the procedures. They develop within the argumentative processes and are the basis of the interpretative activities. These actions involve procedures of a high methodological and metacognitive level: to implement a strategy, to meet a conjecture justification, to find a solution to the problem. Hershkowitz, Schwarz, and Dreyfus defined them as "that actions in which knowledge is used or constructed" [20].

In this study, under the name of epistemic actions, we considered:

1. The actions that correspond to backward reasoning features from the mathematical point of view. These actions are not only cognitive but also involve the qualification provided by mathematical knowledge (Section 2.1).

2. The actions identify as problem-solving heuristics that are inherent to strategy games [18].

3. The actions as defined in AiC theory, the RBC model. These actions give priority of cognitive processes involved in thinking processes (Section 2.3).

\subsection{RBC Model}

AiC theory [16] aims to provide a theoretical and methodological approach for the analysis of epistemic actions. AiC provides researchers with a tool for the analysis of abstraction processes, where abstraction is defined as a vertical reorganization of knowledge [20]. The main aim is to get insight into processes of learning by means of progressive abstraction at the micro-level.

The central theoretical construct of $\mathrm{AiC}$ is the $\mathrm{RBC}$ model $[16,20]$, according to which three types of actions are classified: recognizing (R), building-with (B), and constructing $(\mathrm{C})$.

- $\quad$ Recognizing: Recognizing some previously learned knowledge as relevant for the resolution of the problem.

- $\quad$ Building-with: Combining a set of knowledge with the aim to implement a strategy, to meet a conjecture or justification, or to find a solution to the problem.

- Constructing: Assembling and integrating the previous knowledge with the aim to produce a new construct.

\section{Research Objective and Methodology}

This study formed part of a research experiment designed to examine mathematical reasoning using strategy games. This section describes the experiment, the objective, the context, and the qualitative approach used for its data analysis. 


\subsection{Objective}

The objective of this study was to show how a combination of two approaches, the RBC model and the backward reasoning features, is a suitable tool for the analysis of the cognitive and epistemic actions involved in discovering processes. For this purpose, we pose the following research questions:

1. What epistemic actions, in terms of heuristics use and backward reasoning features, are involved solving the strategy game?

2. What epistemic actions, in terms of chain of thought, are implied in the abstract process of mathematical formula development?

3. How do epistemic actions generate a recurring model in discovering processes following the RBC model?

\subsection{Study Group and Presentation of the Task}

Data were collected in 2018 from 185 Spanish and Italian mathematics and engineering students, aged between 19 and 30. The participants were spread all over the years of academic studies from the first year of bachelor to the last year of $\mathrm{PhD}$. They had different mathematical background regarding solving problems, but they had not received any special training about backward heuristics.

To study the cognitive and epistemic characterization of backward reasoning in strategy games resolution, we proposed 3D Tic-Tac-Toe [22], also known as Qubic in the literature [23]. This is a finite 2 player game with perfect information. It is the 3D version of the Three-in-a-Skate game played on a cubic board of side 4 . The two players choose to adopt two symbols to indicate the position of their tokens in the 64 cubic boxes in which the board is divided. The objective is to horizontally, diagonally, or vertically place 4 marks in a row while trying to block the opponent from doing so. It is a complex game: In 1980, Patashnik solved it through a combination of human expert knowledge and a standard search algorithm, requiring about 1500 computer hour [23].

This game has interesting mathematical properties that we found useful for the research experiment. The board of the $k$-dimensional version $(k>1)$ is a $k$-dimensional hypercube of side $n$, i.e., a $(n, k)$-hypercube. By analyzing the mathematical structure of the game and its winning lines (i.e., all sets of $n$ marks in a row in a $(n, k)$-board), it is possible to build a mathematical formula for the number of its winning lines:

$$
\frac{(n+2)^{k}-n^{k}}{2}
$$

Patashnik [23] and then Golomb and Hales [22] gave a very interesting intuitive proof of (1):

Proof. Given the $(n, k)$-hypercube, the $(n+2, k)$-hypercube embeds the $(n, k)$-hypercube. It is extended one unit farther in each direction in each of the $k$ dimensions. By extending the winning lines of the $(n, k)$-hypercube of a unit in each direction, each of them ends in two border boxes of the $(n+2, k)$-hypercube. Each border box of the enlarged hypercube has only one winning line that pass through it. The $(n+2, k)$-hypercube border boxes are in two-to-one correspondence with the winning lines. There are $(n+2)^{k}-n^{k}$ border boxes in the enlarged hypercube. Thus, there are $\frac{1}{2}\left((n+2)^{k}-n^{k}\right)$ winning lines in the $(n, k)$-hypercube.

For example, the $(5,2)$-hypercube is the extension of the $(3,2)$-hypercube (Three-in-a-Skate board). By drawing the extended winning lines, the following figure is obtained (Figure 1). There are $(3+2)^{2}-3^{2}=16$ boarder boxes in the enlarged hypercube. Thus, there are 8 winning lines in the $(3,2)$-hypercube. 


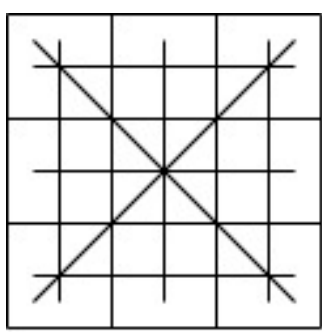

(a)

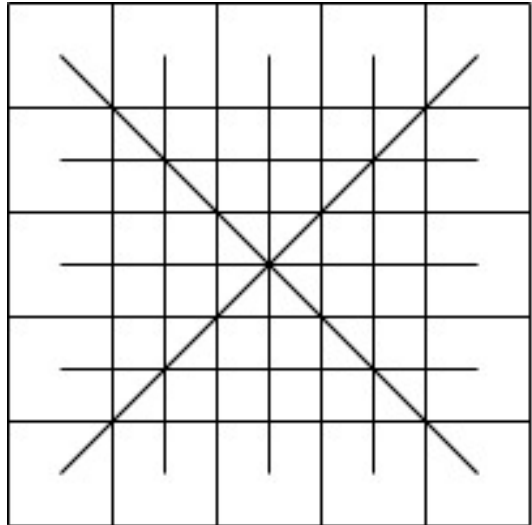

(b)

Figure 1. (a) (3,2)-hypercube with its winning lines and (b) $(5,2)$-hypercube with the $(3,2)$-hypercube extended winning lines.

The board version of the game used for the experiments consisted of a (4,3)-hypercube, as shown in Figure 2. The process consisted of solving the game and finding a relationship between the number of winning lines and the board dimensions. Obtaining the winning strategy was not expected during the experiment. The researchers were interested in the strategies and the reasoning that emerged during the attempt of resolution and the mathematical formulation.

3D Tic-Tac-Toe is the three-dimensional version of the classic Three-in-a-Skate game. The game board is a $4 \times 4 \times 4$ cube.

The game is for two players.

One player uses "crosses" and the other uses "noughts."

The objective is to horizontally, diagonally, or vertically place 4 marks in a row while trying to block the opponent from doing so.
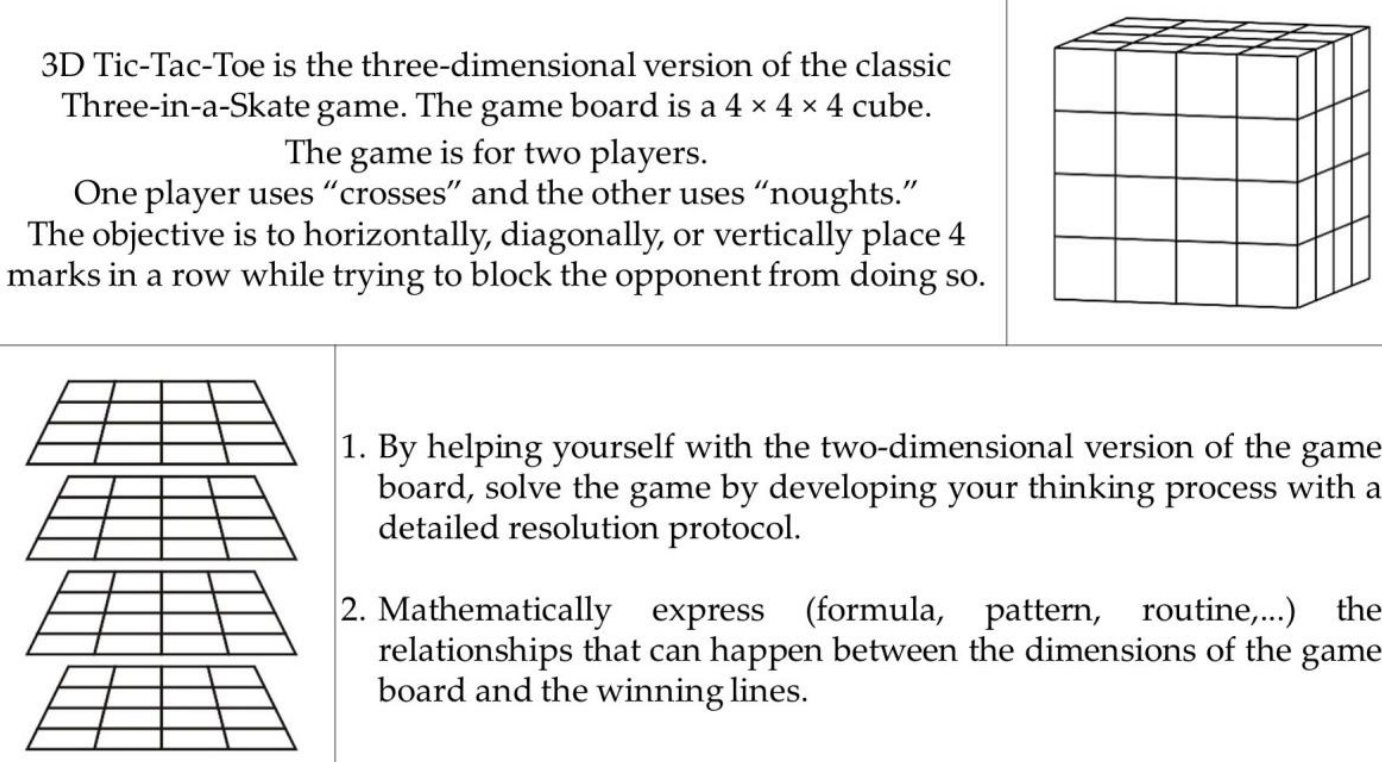

1. By helping yourself with the two-dimensional version of the game board, solve the game by developing your thinking process with a detailed resolution protocol.

2. Mathematically express (formula, pattern, routine,...) the relationships that can happen between the dimensions of the game board and the winning lines.

Figure 2. Task assignment.

The students worked in pairs or alone; we gave them paper and pencil and some empty board to elaborate the task requirements within two hours. Students were also asked to describe their thought processes in the resolution, the difficulties they encountered, and the strategies they would use to solve it. The data were collected through direct observations during the working session, video-recordings of their behaviors, and protocols where students describe their approaches to the task solution.

\subsection{Methodology and Data Analysis}

We followed a qualitative methodology based on design experiment and case study techniques [24]. First, an analysis of the whole group was carried out, and then case studies that considered that 
the students are part of a convenience sample were chosen [25]; these case studies were representatives of their own group class.

The students' resolution protocols were analyzed by dividing them into epistemic actions (see its definition in Section 2.2) and classified using the following categories:

-Backward reasoning features: The epistemic actions that corresponded to backward reasoning were identified depending on which kind of action the students developed: the student does a problem breakdown (D), researches for cause-effect relationships (E), introduces auxiliary elements $(\mathrm{X})$, or formulates the solution object after a series of backward steps (FS). If the action involves forward reasoning, it is not described using this model. In the analysis, a hyphen appears.

-Problem-solving heuristics that are inherent to strategy games.

-RBC model: Each action can be classified as recognizing (R), building-with (B), or constructing (C).

Then, a comparative analysis of the protocols results was developed: Each resolution protocol was observed as a whole, and a recurring pattern was sought along the analysis models.

\subsection{Reliability and Validation of Data Analysis}

The analysis was inductive to explore and identify categories, patterns, and relationships. To ensure the reliability and validation of this study [26], the analysis process also included discussion and comparisons with researchers in order to minimize biases and ensure accuracy. In the analysis, methodologically, the triangulation of sources and judges (three researchers and professors involved in the experimentation) was considered. The combining of different perspectives on the same empirical context allowed us to validate and make the data reliable [25].

\section{Results}

In this section, we show a characterization of the backward reasoning in the whole group, followed by a case study that illustrates the model.

\subsection{Heuristics and Background Mathematical Knowledge in the Group}

Backward reasoning and the relationships with heuristics and background mathematical knowledge in the whole group were studied. We split the students into two groups according to their mathematical background: Students who had a minimum mathematical background, attending the first year of their university courses (Group 1 with 99 students) and students who had a higher mathematical background, such as those who attend the upper courses of the degree or PhDs (Group 2 with 86 students).

Analyzing the students' epistemic actions and processes of thought in which backward reasoning appeared, we identified that backward reasoning was used during the mathematical formula development (see Figure 2) and in other four resolution moments:

- Analyzing geometric properties of winning lines.

- Studying the geometric positions in which most of the lines pass through.

- Searching for the final geometric configuration that leads a player to win the game: This consisted of the configuration in which the winner had two half-finished winning lines at the same time.

This forced the opponent to put a token in a specific position, blocking only one of the two lines.

- Studying the opponent's configuration of winning lines and predicting his/her possible movements.

- Developing the mathematical formula.

According to backward reasoning features (BR column in Table 1) while analyzing winning lines, studying favorable positions, and developing a mathematical formula, the majority of epistemic actions that were involved backward reasoning were classified as breakdowns (D); meanwhile, during searching for the final movements and blocking the opponent, the majority of epistemic actions were classified as the search for cause-effect relationships (E). In all cases, some epistemic actions were classified as the introduction of auxiliary elements $(\mathrm{X})$. 
Table 1. Backward reasoning and academic level groups. BR: backward reasoning; D: breakdown; X: auxiliary elements; E: cause-effect relationship.

\begin{tabular}{ccccc}
\hline & BR & Whole Group & Group 1 & Group 2 \\
\hline Analyzing winning lines & D and X & $55 \%$ & $44 \%$ & $67 \%$ \\
Studying favorable positions & D and X & $81 \%$ & $72 \%$ & $91 \%$ \\
Searching for the final movements & E and X & $56 \%$ & $58 \%$ & $53 \%$ \\
Blocking the opponent & E and X & $75 \%$ & $71 \%$ & $79 \%$ \\
Developing a mathematical formula & D and X & $34 \%$ & $10 \%$ & $62 \%$ \\
\hline
\end{tabular}

From a heuristic point of view, we identified six heuristics or strategies that students developed during the resolution (Table 2). Among them, those based on backward reasoning are called backtracking heuristics.

Table 2. Heuristics developed divided by academic level groups.

\begin{tabular}{cccc}
\hline Heuristics & Whole Group & Group 1 & Group 2 \\
\hline Making a systematic study of all geometric positions & $81 \%$ & $70 \%$ & $93 \%$ \\
Using a mathematical language & $56 \%$ & $31 \%$ & $85 \%$ \\
Visualizing the geometrical properties of 3D board & $43 \%$ & $29 \%$ & $58 \%$ \\
Reducing geometrically the board size & $56 \%$ & $55 \%$ & $58 \%$ \\
Making diagrams, drawings, graphic representations & $73 \%$ & $67 \%$ & $80 \%$ \\
Using backtracking heuristics & $80 \%$ & $76 \%$ & $85 \%$ \\
\hline
\end{tabular}

The data indicated that the mathematical background affected the resolution of the task. Only 63 students (10 students from Group 1 and 53 from Group 2) developed the mathematical formula (34\%). To do it, they analyzed the geometric properties of the board and used their algebraic knowledge. Its development has a higher percentage of realization in Group $2(62 \%)$ than in Group 1 $(10 \%)$. A similar result appeared in the heuristics analysis when using a mathematical language, visualizing the geometrical properties of 3D board, and geometrically reducing the board size, three heuristic processes in which background mathematical knowledge is deeply involved.

\subsection{Patterns of Epistemic Actions}

The whole group analysis showed that $80 \%$ of the students used backtracking heuristics (see Table 2), and five moments in which backward reasoning was developed could be identified in their thought processes (Table 1). The first four moments belonged to the resolution discovering phase. Regarding the mathematical formula development, it was split in two phases: discovering and verifying. In the first one, the students elaborated the formula, and they justified it in the second. Backward reasoning occurred only in discovering phases.

The use of the RBC model for the analysis of the protocols allowed us to identify two common recurring sequences of epistemic actions: $\mathrm{B}-\mathrm{R}-\mathrm{C}$ and $\mathrm{B}-\mathrm{C}$ sequences.

When $\mathrm{B}-\mathrm{R}-\mathrm{C}$ sequences took place, the students' process of thought proceed in this manner:

1. They did an exploration/manipulation of the elements/data/concepts given by the problem.

2. They recognize an element/concept useful for the resolution.

3. They manipulated the objects that appeared in previous point in order to build a new concept.

When B-C sequences occurred, the students' processes of thought proceed in this way:

1. They did an exploration/manipulation of the elements/data/concepts given by the problem.

2. They manipulated the objects given by the problem in order to build a new concept.

$\mathrm{B}-\mathrm{R}-\mathrm{C}$ sequences characterized all the backward reasoning moments, while $\mathrm{B}-\mathrm{C}$ sequences appeared alongside the first ones in developing a mathematical formula. In fact, $\mathrm{B}-\mathrm{R}-\mathrm{C}$ sequences 
were produced when students constructed the formula, while B-C sequences were produced when they manipulated it to obtain the final expression. B-C sequences also took place in the mathematical formula verifying phase where backward reasoning did not occur. Table 3 summarizes the recurring pattern of reasoning sequences according to the phases for the 185 students.

Table 3. Pattern of reasoning sequences in the whole group according to the phases. C: constructing.

\begin{tabular}{cccc}
\hline & Whole Group & Discovering Phase & Verifying Phase \\
\hline Analyzing winning lines & $55 \%$ & B-R-C & - \\
Studying favorable positions & $81 \%$ & B-R-C & - \\
Searching for the final movements & $56 \%$ & B-R-C & - \\
Blocking the opponent & $75 \%$ & B-R-C & - \\
Developing a mathematical formula & $34 \%$ & B-R-C and B-C & B-C \\
\hline
\end{tabular}

Finally, in abstraction processes, a recurring behavior (see Figure 3) was identified in the reasoning movements throughout three different representational contexts: one related to the game, the second in which mathematical particularization was developed, and the third in which mathematical concepts were generalized. In the discovering phase, the reasoning developed back and forth through the contexts, while in the verifying phase, it had a more linear trend. In the discovering phase, the analysis showed that, since the final abstraction concept was unknown, the epistemic actions content changed according to the three contexts. Through processes of exploration and progressions-regressions between contexts, the students constructed and understood the mathematical structure underlying the concept. In the verifying phase, since the final concept was known, the reasoning ran through the concept mathematical structure which was previously built.
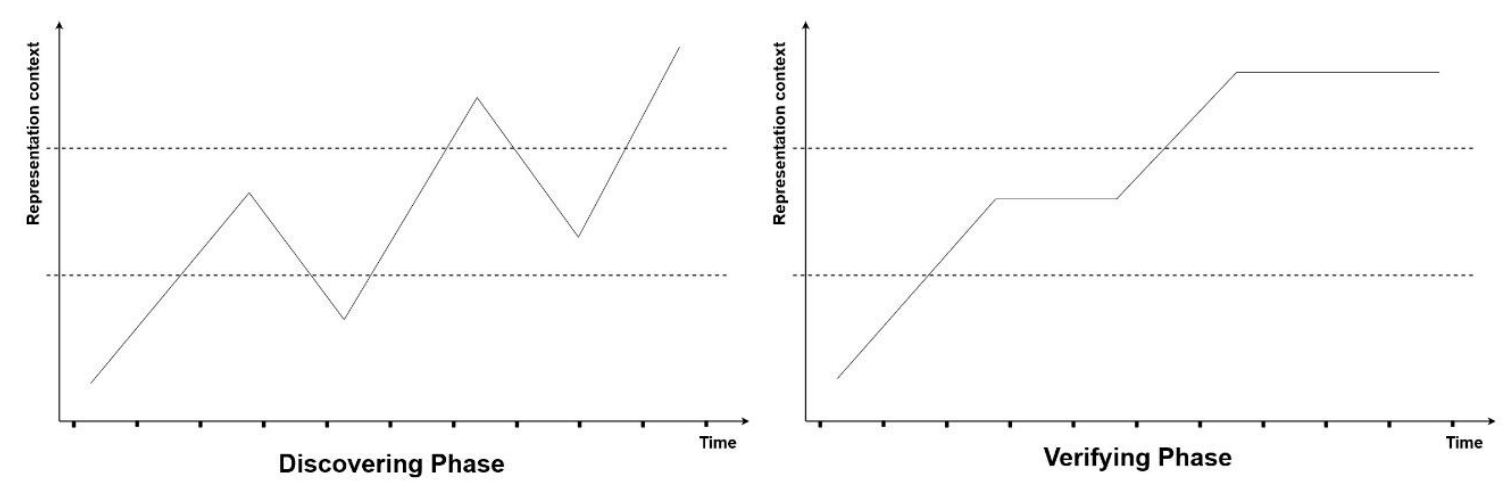

Figure 3. Backward reasoning behavior in discovering and verifying phases.

In Section 4.3, through a case study, we illustrate how the microscopic analysis was carried out to reach these results. The reasoning trends in backward reasoning moments are shown through a significant example concerning the mathematical formula development and its justification.

\subsection{Epistemic Actions Model: Case Study}

In this section, we illustrate how the epistemic actions sequences can concretely develop through a descriptive case-study [24]. We show the protocol of Student-A: He was key informant of the PhD students' group (consisting of eight students). He is an expert student who solved the task by investigating the mathematical relationships that were at the basis of the game. He wrote a highly elaborate protocol, structuring it according to 35 points. We identified some breaking elements in his reasoning sequences that triggered his processes of thought. Two excerpts of his protocol are shown. They belonged to a discovering phase (mathematical formula development) and a verifying phase (formula verification) of his resolution. 
The student began the game resolution by solving the $2 \mathrm{D}$ version of the game (Three-in-a-Skate game). First, he played while trying to remember the winning strategy, and then he started to calculate the number of winning lines. Later, he moved on to the 3D version of the game, $(4,3)$-hypercube, where he continued to reason out the number of winning lines until he obtained a general formula. Then, he showed that the formula he had found was valid for any cube of (n,d) dimensions, and, finally, he reasoned again about the winning strategy, this time for the 3D case.

The first excerpt (Figure 4) refers to the discovering process that led the student to create the mathematical formula. Backward reasoning strongly characterized this process.

12. I decide to move on to the 3D case. The previous strategy suggests that I count lines. I make a few drawings to test. There are 10 lines in each plan parallel to the axes, and there are 12 planes parallel to the axes. I lack the "diagonal lines" as in the example. They seem more complicated.

13. I am starting to do numerology: $10=4 \cdot 2+2$ which is broken down as the number of tokens per dimension of the plane plus two diagonals. Will it be general?

14. I realize that $12=4 \cdot 3$, which seems to follow the previous pattern. I hope. It looks like a nice combinatorial problem.

15. It reminds me of geometry calculations on finite fields. I think about shooting over there, but I realize that there are cyclic lines that come out on one side and appear on the opposite side. These movements are not allowed. I could rule them out, but it seems too complicated. I abandon this strategy.

16. I think of a recursive pattern. I guess $\mathrm{n}$ pieces in $\mathrm{d}$ dimensions (the usual case is $(n, d)=$ $(3,2)$, and this is $(n, d)=(4,3))$. Maybe the number of straight lines follows a pattern.

$$
L(n, d)=\operatorname{cnt}(n, d) \cdot L(n, d-1)+\text { Diagonals * }
$$

17. The factor $[\operatorname{cnt}(n, d)]$ must be the number of planes parallel to the axes. As in the previous case, these have to be $n d$, so I refine my formula to:

$$
L(n, d)=n d \cdot L(n, d-1)+\text { Diagonals }
$$

18. Diagonals do not seem that simple. I start to play with the example of the cube and the plane. They seem to join opposite vertices of opposite faces. Will it be general?

19. I calculate that a hypercube has $2^{d}$ vertices, which gives me two faces with $2^{d-1}$ vertices. Thus, if my previous observation is correct, the formula is:

$$
L(n, d)=n d \cdot L(n, d-1)+2^{d-1}
$$

* Student-A uses $L(n, d)$ to indicate the number of winning lines depending on $\mathrm{n}$ and $\mathrm{d}$ values, and he uses $\operatorname{cnt}(n, d)$ to indicate a factor depending on $\mathrm{n}$ and $\mathrm{d}$ value.

Figure 4. First excerpt of Student-A protocol: formula development phase.

The student's first objective was to count the winning lines on the board. To do so, he divided the board into planes and counted the winning lines on each of them. Then, he started to think about the found number. Breaking it down, he tried to identify some game elements (number of tokens for each winning line, size, number of diagonals) in its formulation. Later, he analyzed and broke down each plane in the same way. At this point, he introduced a recursive auxiliary pattern and conjectured the existence of a general recursive formula that related the number of winning lines with the board size. Finally, by analyzing the raw formula (line 16), he looked for a mathematical expression for each part of it. Thus, he obtained the general recursive formula. The winning lines analysis that led to the conjecture was a basic step for the formula development.

By analyzing the excerpt, it was possible to identify different epistemic actions performed by the student. Each of them could be characterized according to the backward reasoning features and the RBC model. In the following table (Table 4), the excerpt analysis is summarized: the epistemic actions, the features of backward reasoning (BR column), and the RBC model classification (RBC column) are identified. Where backward reasoning did not take place, a hyphen is shown. 
Table 4. First excerpt of Student-A protocol: analysis of epistemic actions. RBC: recognizing, building-with, and constructing; FS: backward steps.

\begin{tabular}{cccc}
\hline Protocols & Epistemic Action & BR & RBC \\
\hline \multirow{2}{*}{12} & Splitting the board into planes & $\mathrm{D}$ & $\mathrm{B}$ \\
& Counting the winning lines in each plan & $\mathrm{D}$ & $\mathrm{B}$ \\
& Grouping winning lines into a scheme & $\mathrm{X}$ & $\mathrm{R}$ \\
\hline \multirow{2}{*}{13} & Mathematically breaking down a number & $\mathrm{D}$ & $\mathrm{B}$ \\
& Identifying each element of the decomposition & $\mathrm{X}$ & $\mathrm{C}$ \\
\hline 14 & Mathematically breaking down a number & $\mathrm{D}$ & $\mathrm{B}$ \\
\hline 15 & Analogy/break motion & - & $\mathrm{R}-\mathrm{B}$ \\
\hline \multirow{2}{*}{16} & Introducing a recursive pattern & $\mathrm{X}$ & $\mathrm{R}$ \\
& Conjecturing: general recursive formula & $\mathrm{FS}$ & $\mathrm{C}$ \\
\hline 17 & Breaking down the formula into its elements & $\mathrm{D}$ & $\mathrm{B}$ \\
& Analyzing the factor element & $\mathrm{D}$ & $\mathrm{B}$ \\
\hline \multirow{2}{*}{19} & Representing the factor in relation to the board dimensions & $\mathrm{D}$ & $\mathrm{C}$ \\
\hline & Analyzing the diagonal element & $\mathrm{D}$ & $\mathrm{B}$ \\
\hline
\end{tabular}

From backward reasoning point of view, the student broke down the problem and inserted auxiliary elements in an alternating way. He did it to conjecture the existence of a general recursive formula and to mathematically represent it. The actions involved in breakdown moments (D) supposed a longer time of realization, while the introduction of auxiliary elements $(X)$ was instantaneous. Line 15 corresponds to a structural analogy; it broke Student-A's chain of thought, thus triggering the following epistemic actions. From the RBC model point of view, a certain regularity was noticed (see last column in Table 4): Two B-R-C sequences (B-R-C-B-R-C) characterized the formulation of the conjecture, while two $\mathrm{B}-\mathrm{C}$ sequences $(\mathrm{B}-\mathrm{C}-\mathrm{B}-\mathrm{C})$ characterized the general formula expression.

Recognizing actions changes the nature of the reasoning. Through them, the student introduced new elements in the resolution, whether they were specific to the problem (the scheme) or mathematical constructs that were structurally analogous (the recursive pattern). These actions were of two kinds: In the former, the student created a new construct processing already encountered knowledge in the resolution, and in the latter, the new construct emerged from a structural analogy (line 15). During this analogy, the student remembered geometrical concepts on finite fields that helped him identify patterns.

When the conjecture was formulated and the raw mathematical formula had been obtained, the reasoning changed. The student focused on the conjectured formula and analyzed it element by element until the desired mathematical expression was obtained. The reasoning proceeded within $\mathrm{B}-\mathrm{C}$ sequences. No recognizing actions appeared, as the reasoning developed through the elaboration of notions that were already clear in his mind. The breakdown feature that portrayed this process was not continuous, but was interspersed with moments of forward reasoning. In fact, the student derived the expression of the factor and the diagonals from the notions previously learned in the resolution and then made them explicit in a forward way.

The second excerpt (Figure 5) refers to the verifying phase, the moment in which the student checked the just obtained mathematical formula. Backward reasoning was absent in this part, which was characterized only by synthesis processes. 
20. I try it on low cases. It works. I think about proving it by induction, but I still have the problem of diagonals. I abandon the idea.

21. I try with $n=3, d=4$ to check the formula. I have the problem of representing it, but I decide to follow the representation of the previous exercise and arrange 3 juxtaposed cubes. I realize that what I am actually doing is setting the first coordinate and moving the others. Is this maybe the recursive pattern?

22. I realize that the key is which is the direction vector of the line. If it has a zero, that coordinate remains fixed; in reality, it is a line of the recursive case.

23. Aha! That seems to be the way to proceed to the end. I realize that the direction vector has to have the form $v=\left(\varepsilon_{1}, \ldots, \varepsilon_{d}\right)$ with $\varepsilon_{i}= \pm 1,0$. That seems to be the key to count the winning lines.

24. If any $\varepsilon_{i}=0$, then the line belongs to a parallel hyperplane. There are $d$ positions to put the zero, and, in that case, it can start at any of the $n$ hyperplanes. That justifies the $n d$. $L(n, d-1)$. Perfect.

25. If all $\varepsilon_{i}= \pm 1$, then I realize that they have to go from vertex to vertex. I also realize that the original vertex is fixed by $v$, since it is $\left(\delta_{1}, \ldots, \delta_{d}\right)$ with $\delta_{i}=\frac{n-n \varepsilon_{i}}{2}$. That would give me $2^{d}$ diagonals, which is as many as $v$ possibilities.

26. It does not work even in low cases. I realize that $v$ and $-v$ give the same straight line. Light. There is an action of $\mathbb{Z}_{2}$ and that gives me the desired $2^{d-1}$ diagonals. Proven conjecture.

Figure 5. Second excerpt of Student-A protocol: formula verification phase.

The student started to test the formula using small $\mathrm{n}$ and $\mathrm{d}$ values, so that, with his acquired game knowledge and counting the lines case by case, he could easily verify the formula. Then, he thought about proving the correctness of the general formula. He began thinking about proving it by induction, but he had difficulties representing diagonal values and abandoned the idea. Later, reasoning about the $4 \mathrm{D}$ case, Student-A recognized an analogy in the analytical structure of the problem and associated each winning line to a direction vector. Through the winning lines, represented as direction vectors, the student was able to rigorously construct the mathematical formula found previously. He made a continuous control on his actions and realized that there was something wrong in the reasoning (line 26) He managed to overcome his difficulty thanks to the introduction of another element from previous knowledge: the action of $\mathbb{Z}_{2}$.

As in the previous case, the different epistemic actions performed by the student were identified and classified according to the backward reasoning features and the RBC model. In the following table (Table 5), the excerpt analysis is summarized.

Table 5. Second excerpt of Student-A protocol: analysis of epistemic actions.

\begin{tabular}{|c|c|c|c|}
\hline Protocols & Epistemic Action & BR & RBC \\
\hline \multirow{2}{*}{20} & Checking the formula & - & $\mathrm{B}$ \\
\hline & Identifying possible proof path & - & $\mathrm{R}$ \\
\hline \multirow{2}{*}{21} & Checking the formula in $4 \mathrm{D}$ case & - & $\mathrm{B}$ \\
\hline & Representing the $4 \mathrm{D}$ case & - & $\mathrm{C}$ \\
\hline \multirow{2}{*}{22} & Identifying a structural analogy with analytical representation & - & $\mathrm{R}$ \\
\hline & Reasoning about possible coordinates & - & $\mathrm{B}$ \\
\hline 23 & Identifying the direction vector & - & $\mathrm{C}$ \\
\hline \multirow{2}{*}{24} & Analyzing $\varepsilon_{i}=0$ cases Introducing a recursive pattern & - & B \\
\hline & Justifying “ $n d \cdot L(n, d-1)$ ” & - & $\mathrm{C}$ \\
\hline \multirow{2}{*}{25} & Analyzing $\varepsilon_{i}= \pm 1$ cases & - & B \\
\hline & Constructing diagonals value: $2^{d}$ & - & $\mathrm{C}$ \\
\hline \multirow{2}{*}{26} & Identifying an action of $\mathbb{Z}_{2}$ & - & $\mathrm{R}$ \\
\hline & Constructing diagonals value: $2^{d-1}$ & - & $\mathrm{C}$ \\
\hline
\end{tabular}


As anticipated before, backward reasoning was absent in this part. The excerpt was characterized by a series of B-C sequences. They were interrupted in lines 20, 22, and 26 by recognizing actions. There were two crucial structural analogies: The analytical representation led to the justification of the mathematical formula, and the action of $\mathbb{Z}_{2}$ allowed for the identification and the overcoming of difficulties that emerged in the resolution. The reasoning developed through the elaboration of notions deriving from the game resolution experience and the student's background. In the following diagram (Figure 6), the sequences of actions identified in the previous excerpts are highlighted.

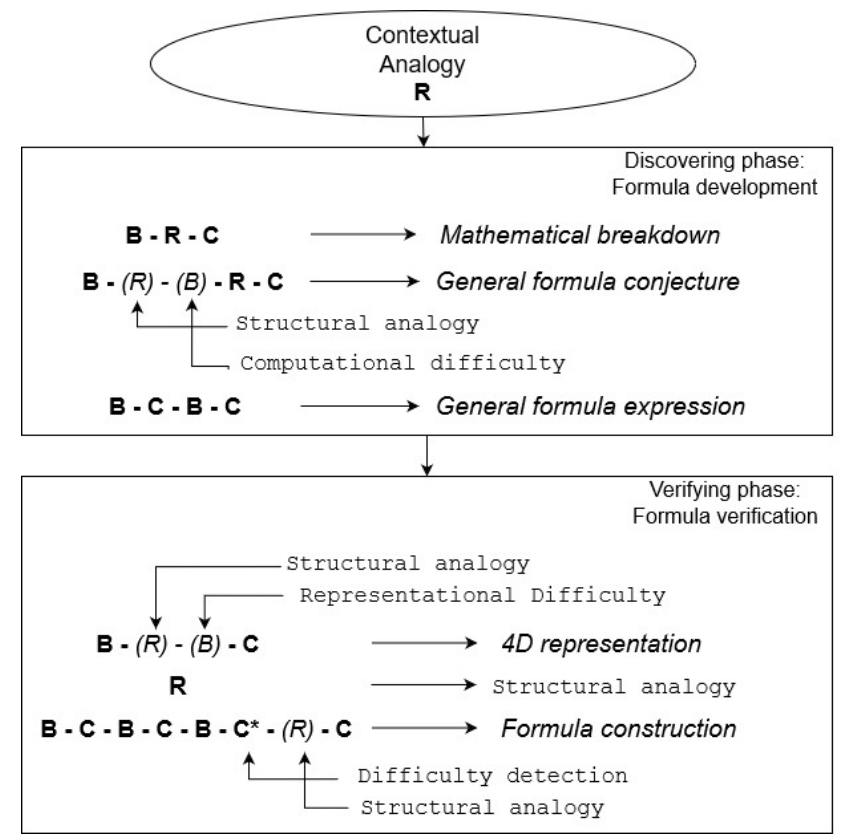

Figure 6. First and second excerpt RBC flow (Student-A resolution).

If we look at the whole process of the first excerpt (Figure 7), we can see how the student passed through different contexts in order to achieve the general mathematical formulation. He began working within the strict context of the proposed game (informal), and then he moved to a mathematical context to interpret the example; afterwards, he went forward and explained the game in a more general mathematical context. The transition between the three contexts happened with a complex back and forth process, where the different contexts were repeatedly activated. The analogies that came into play are highlighted.

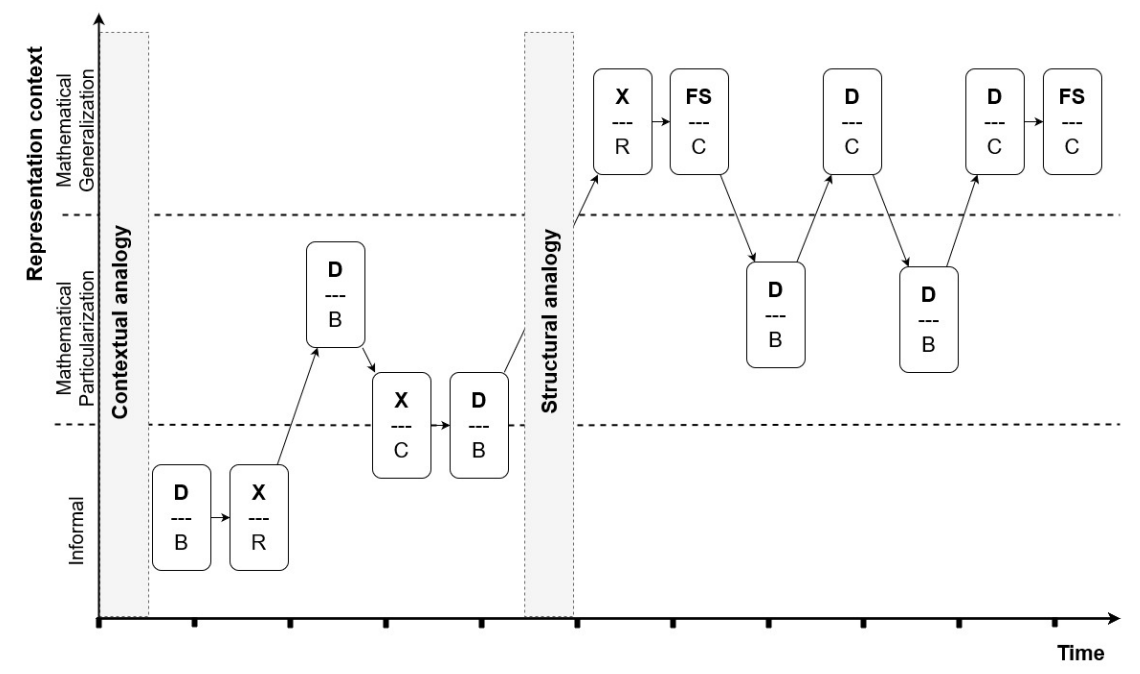

Figure 7. Pattern in epistemic actions and context (first excerpt diagram). 
Likewise, if we observe the whole process of the second excerpt (Figure 8), we can see how the student also passed through different contexts in order to achieve the general mathematical formulation and to justify his precedent achievement also in this case. The progression was linear, from the informal context to the mathematical generalization passing through the mathematical particularization. Additionally in this case, the analogies appear in the diagram.

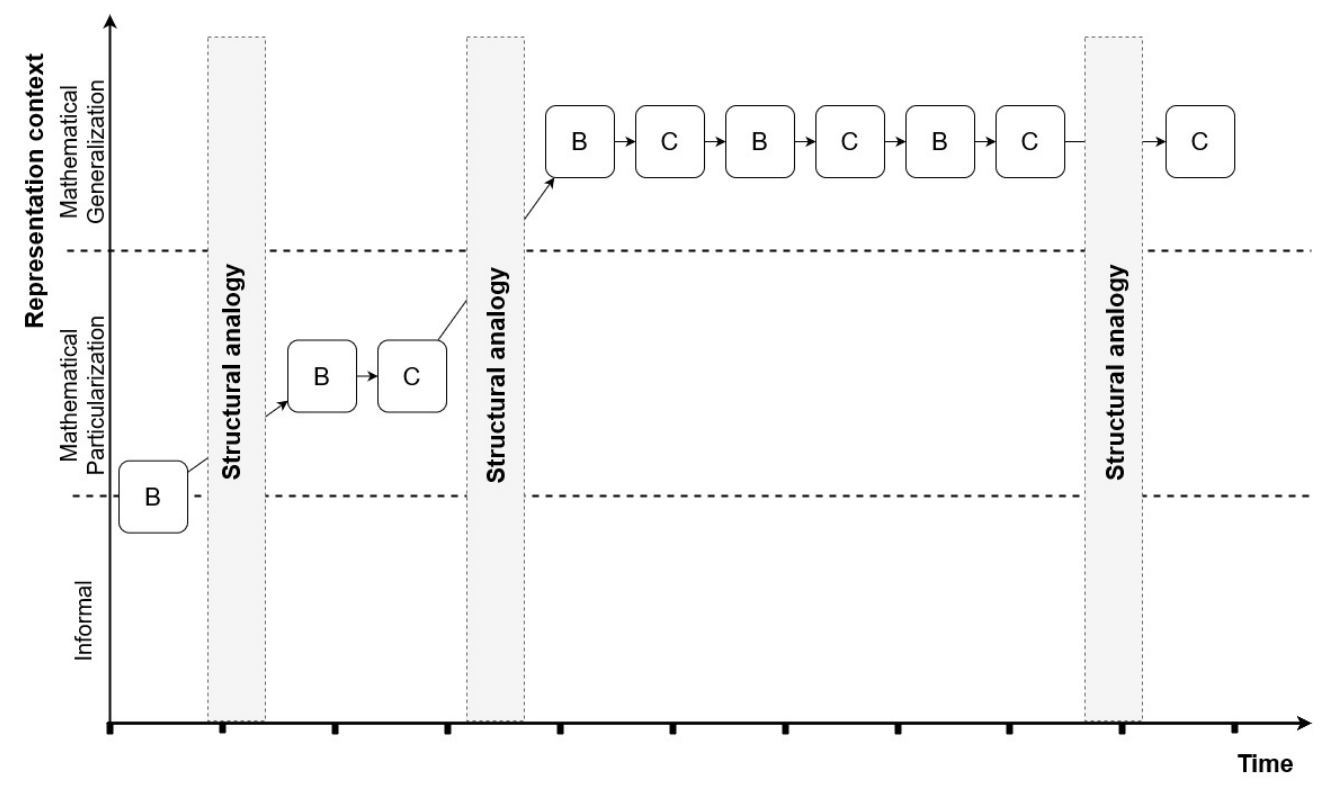

Figure 8. Pattern in epistemic actions and context (second excerpt diagram).

In both cases, analogies occurred and broke the chains of thought. They were crucial because they triggered the subsequent actions, leading Student-A to construct more general mathematical concepts.

\section{Discussion and Conclusions}

The main objective of this study was to show the combination of two approaches-the RBC model with the mathematical backward reasoning features-as a tool for the analysis of the cognitive and epistemic actions involved in discovering processes. Three research questions were established to analyze this combination. The major findings were: (1) There were significant connections between epistemic actions in terms of heuristics and mathematical knowledge background; (2) in discovering phases in which backward reasoning was involved, B-R-C and B-C sequences appeared; in verifying phases, only B-C sequences appeared; (3) in abstraction processes, the RBC flow and reasoning movements throughout three different representational contexts were defined, all of which consented to detect breaking elements and moments of non-linearity.

These findings allowed us to confirm the usefulness of the analysis categories offered by the model of AiC theory as a tool for the analysis of the epistemic actions at a tiny level. The study shows that, for a more global analysis of the thoughts and the long lasting learning processes, the RBC model has to be complemented with other categories that allow for the identification of the movements between the abstraction contexts and the breaking elements. These categories are the backward reasoning mathematical features.

Regarding the first research question, the relations between the use of heuristics and mathematical knowledge (see Tables 1 and 2), students with a higher mathematical background developed backward reasoning moments and heuristics strategies in which mathematical knowledge was strongly involved. The differences were significant, with more than $50 \%$ participants between groups using a mathematical language and developing a mathematical formula, as well as in the identification of properties of a 3D board, in which the difference was $29 \%$ participants. 
With regard to the second research question, two common recurring sequences of epistemic actions in backward reasoning moments, B-R-C and B-C sequences, were identified (see Table 3 ). In discovering phases, the B-R-C sequences corresponded to inquiry moments, while the B-C sequences corresponded to processes of construction of mathematical concepts. The B-R-C sequence led to students having a moment of exploration at the beginning of the process (building-with); then, they recognized a concept or a structure, often getting it from their background, which could be useful for the resolution; finally, they achieved the construction of a new concept. Backward reasoning developed during the breakdown of the problem and was characterized by the introduction of auxiliary elements that were related to the recognizing actions. They took place through the following students' backward reasoning moments: analyzing winning lines processes (55\% of participants), studying favorable position (81\% of participants), searching for the final movements (56\% of participants), blocking the opponent (75\% of participants), and developing a mathematical formula (34\% participants). Instead, when the backward reasoning developed in correspondence of manipulative processes, B-C sequences appeared. This was the case of the discovering phase of developing a mathematical formula (34\% of participants). In these cases, backward reasoning was not continuous, as it was instead interrupted by moments of forward reasoning. While backward moments corresponded to building-with actions, forward moments corresponded to constructing actions. The solver transformed known notions in a backward way, and then he used them to progressively build new concepts. In verifying phases, instead, only forward reasoning and B-C chains were developed. Polya [18] called "looking back" the phase where the results, which have been obtained in the previous ones, are verified. It corresponds to the last part of the problem solving. Deductive logical inferences are developed in order to reverse the passages of the analysis and get a justification for the resolution. In the proposed game, the mathematical formula was constructed with step-by-step reasoning about known knowledge. B-C sequences were sometimes interrupted (as in the case study example; see the second excerpt) by recognizing actions that allowed for the introduction of structural analogies to detect difficulties and errors and then overcoming them.

Regarding the third question, the data showed that the identification of epistemic actions generated a recurring model of the abstraction processes in discovering phases (see Figure 3 ). It is clearly exemplified in Figure 4 (excerpt 1) and Figure 7 of the case study. This was not linear from the informal context to mathematical generalization. It proceeded with back and forth movements between the mathematical representation contexts until the solution was elaborated in a general form. These types of movements have already been pointed out by Gómez-Chacón et al. [27] and have been identified thanks to the extension of the RBC model with the backward reasoning features that allows for the grasping of the overall reasoning process. Backward movements towards the mathematical particularization context facilitated transitions between the other contexts. The transition through it was necessary to firstly develop a system of signs and secondly to understand the interaction among the different involved elements, identified with the problem breakdown. The use of algebraic and logical properties allowed them to "translate" game properties into general abstract mathematical elements. The backward reasoning helped to connect simpler components with the complex ones, investigating the basic principles and generalizing them. On the other hand, in verifying phases, the reasoning proceeded linearly from the informal/particular mathematical context to the general one. This way of proceeding is typical of forward reasoning. Starting from the premises, or from the basis of the problem, the solver reached the solution through deductive logical steps.

The data of the Student-A case study showed (Section 4.3) some hints that helped to clarify the general reasoning behavior and the role of breaking elements in his thought processes. When the mathematical structure was unknown, the path to reach the sought abstraction elements was not linear, and backward ways of thinking were used to reach the goal, while if the concepts underlying the sought elements were clear, the reasoning proceeded in a linear way. Thus, it required inductive, abductive, and backward reasoning, not only forward reasoning [3]. 
To understand mathematical concepts, it is necessary to comprehend the entire structure and all the thinking progression necessary for its construction [28].

In discovering phases, the incorporation of mathematical backward reasoning categories allowed the student to identify breaking elements and how they trigger the Student-A's construction processes. The analogies in these phases could not be determined only according to the RBC model. In this case, it was necessary to identify elements of the constructive epistemic action processes produced in the long term. A further analysis was required since the RBC model alone was not able to describe the phenomenon from a holistic perspective (see Figure 7). In the cognitive route between the concrete and the abstract, the analogy processes represented a break in the chain of thought that allowed for the advancement towards the construction of ideas.

The above results leave open questions that could be the subject of future considerations, both from the point of view of research and of mathematics and engineering teaching at university level. The variable worked in the RBC model can significantly predict students' performance in backward reasoning in terms of: (a) identifying what backward heuristics are important in a problem solving situation, (b) using some characteristics of backward reasoning (mathematical processes, skills, and the person's ability), and (c) making connections between broader features of backward reasoning to overcome specific difficulties.

Two final observations. First, the backward approach that we analyzed above is at the basis of many programs for automatic theorem proving in elementary geometry, e.g., those which use the Grobner-Buchberger algorithm [29]. The algebraic varieties, which are the geometric counterpart of the algebraic symbolism incorporated into the software, are the natural model of a dynamic logic, where the so-called method of resolution used there means looking for a new economic hypothesis, which allows one to restrict the domain of validity of a supposed conjecture in order to make this really true, exactly in the same way that we pictured the RBC flow above [30] (pp. 280-296). Second, further studies could more deeply explore the subjects' profiles and the individual differences by using data mining tools. For such studies, the identified sequences of epistemic chains of actions could be considered.

Author Contributions: Conceptualization, M.B., I.M.G.-C., and F.A.; methodology, M.B., I.M.G.-C., and F.A.; design experiment and data collection, M.B. and I.M.G.-C.; validation, M.B., I.M.G.-C., and F.A.; formal analysis, M.B.; investigation, M.B., I.M.G.-C., and F.A.; writing-review and editing, M.B., I.M.G.-C., and F.A.; supervision, I.M.G.-C. and F.A.; project administration, I.M.G.-C.; funding acquisition, M.B. and I.M.G.-C. All authors have read and agreed to the published version of the manuscript.

Funding: This study was funded by IMI Institute special action grant, Programme INVEDUMAT_uni (Research in Mathematical Education at University Level) and Innova-Docencia-UCM-252-2019 in Spain and Science Doctoral School of l'Università degli Studi di Torino e il Politecnico di Torino in Italy.

Conflicts of Interest: The authors declare no conflict of interest.

\section{References}

1. Hintikka, J.; Remes, U. The Method of Analysis: Its Geometrical Origin and Its General Significance; Reidel: Dodrecht, The Netherlands, 1974.

2. Lakatos, I. Mathematics, Science and Epistemology; Worrall, J., Currie, G., Eds.; Cambridge University Press: Cambridge, UK, 1978.

3. Hintikka, J. Inquiry as Inquiry: A logic of Scientific Discovery; Springer Science \& Business Media: Dordrecht, The Netherlands, 1999.

4. Grabczewski, K. Meta-Learning in Decision Tree Induction; Springer: Cham, Switzerland, 2014.

5. Dias, J.; Paiva, A. Feeling and reasoning: A computational model for emotional characters. In Portuguese Conference on Artificial Intelligence; Springer: Heidelberg, Germany, 2005; pp. 127-140.

6. Marsella, S.C.; Gratch, J. EMA: A process model of appraisal dynamics. Cogn. Syst. Res. 2009, 10, 70-90. [CrossRef]

7. Picard, R.W. Affective Computing; MIT Press: Cambridge, MA, USA, 1997. 
8. Trivino, G.; Alvarez-Alvarez, A.; Bailador, G. Application of the computational theory of perceptions to human gait pattern recognition. Pattern Recognit. 2010, 43, 2572-2581. [CrossRef]

9. Hintikka, J.; Halonen, I.; Mutanen, A. Interrogative logic as a general theory of reasoning. In Handbook of Practical Reasoning; Johnson, R.H., Woods, J., Eds.; Kluwer Academic: Dordrecht, The Netherlands, 2002; pp. 295-337.

10. Antonini, S. Generating examples: Focus on processes. ZDM Math. Educ. 2011, 43, 205-217. [CrossRef]

11. Peckhaus, V. Regressive Analysis. Hist. Philos. Log. Anal. 2002, 5, 97-110.

12. Barbero, M.; Gómez-Chacón, I.M. Analyzing Regressive reasoning at university level. In Proceedings of the Second Conference of the International Network for Didactic Research in University Mathematics (INDRUM), Kristiansand, Norway, 5-7 April 2018; Durand-Guerrier, V., Hochmuth, R., Goodchild, S., Hogstad, N.M., Eds.; University of Agder \& INDRUM: Kristiansand, Norway, 2018; pp. 204-213.

13. Gómez-Chacón, I.M.; Barbero, M. Situation Specific-Skills Working Backward Reasoning: The Student's Perceptions and Affect Dimension. In Proceedings of the Eleventh Congress of the European Society for Research in Mathematics Education (CERME11), Utrecht, The Netherlands, 6-10 February 2019; Jankvist, U.T., Van den Heuvel-Panhuizen, M., Veldhuis, M., Eds.; Freudenthal Group \& Freudenthal Institute, Utrecht University and ERME: Utrecht, The Netherlands, 2019; pp. 1427-1434.

14. Gómez-Chacón, I.M. Emotions and heuristics: The state of perplexity in mathematics. ZDM Math. Educ. 2017, 49, 323-338. [CrossRef]

15. Hilbert, D.; Cohn-Vossen, S. Geometry and the Imagination; Translation from the German original of 1932; AMS Chelsea: New York, NY, USA, 1952.

16. Dreyfus, T.; Kydron, I. Introduction to Abstraction in Context (AiC). In Networking of Theories as a Research Practice in Mathematics Education; Bikner-Ahsbahs, A., Prediger, S., Eds.; Springer: Heidelberg, Germany, 2014; pp. 85-96.

17. Beaney, M. Analysis. The Stanford Encyclopedia of Philosophy. In Metaphysics Research; Zalta, E.N., Ed.; Stanford University Lab: Stanford, CA, USA, 2018.

18. Polya, G. How to Solve It: A New Aspect of Mathematical Method; Doubleday: New York, NY, USA, 1945.

19. Kirsh, D.; Maglio, P. On distinguishing epistemic from pragmatic action. Cogn. Sci. 1994, 18, 513-549. [CrossRef]

20. Hershkowitz, R.; Schwarz, B.B.; Dreyfus, T. Abstraction in context: Epistemic actions. J. Res. Math. Educ. 2001, 32, 195-222. [CrossRef]

21. Pontecorvo, C.; Girardet, H. Arguing and reasoning in understanding historical topics. Cogn. Instr. 1993, 11, 365-395. [CrossRef]

22. Golomb, S.W.; Hales, A.W. Hypercube Tic-Tac-Toe. In More Games of No Chance, Proceeding of the MSRI Workshop on Combinatorial Games, Berkeley, CA, USA, 24-28 July 2000; Nowakowski, R.J., Ed.; MSRI Publications, Cambridge University Press: Cambridge, UK, 2002; Volume 42, pp. 167-182.

23. Patashnik, O. Qubic: $4 \times 4 \times 4$ Tic-Tac-Toe. Math. Mag. 1980, 53, 202-216.

24. Bassey, M. Case Study Research in Educational Settings; Open University Press: Buckingham, UK, 1999.

25. Gliner, J.A.; Morgan, G.A.; Leech, N.L. Sampling and Introduction to External Validity. In Research Methods in Applied Setting: An Integrated Approach to Design and Analysis; Gliner, J.A., Morgan, G.A., Leech, N.L., Eds.; Routledge Taylor \& Francis Group: New York, NY, USA, 2009; pp. 115-133.

26. Lincoln, Y.S.; Guba, E.G. Naturalistic Inquiry; Sage Publications: Newbury Park, CA, USA, 1985.

27. Gómez-Chacón, I.M.; Romero Albaladejo, I.M.; del Mar García López, M. Zig-zagging in geometrical reasoning in technological collaborative environments: A Mathematical Working Space-framed study concerning cognition and affect. ZDM Math. Educ. 2016, 48, 909-924. [CrossRef]

28. Tall, D. Advanced Mathematical Thinking; Springer: Dordrecht, The Netherlands, 2002.

29. Chou, S.C. Mechanical Geometry Theorem Proving; Reidel: Dordrecht, The Netherlands, 1988.

30. Cox, D.; Little, J.; O'Shea, D. Ideals, Varieties and Algorithms; Springer: Berlin, Germany, 1992.

(C) 2020 by the authors. Licensee MDPI, Basel, Switzerland. This article is an open access article distributed under the terms and conditions of the Creative Commons Attribution (CC BY) license (http://creativecommons.org/licenses/by/4.0/). 